Cahiers de recherches médiévales

Journal of medieval studies

3 | 1997

Espace vécu, mesuré, imaginé

\title{
Temps et espace du voyage chez Liutprand de Crémone
}

\section{Michel Balard}

\section{(2) OpenEdition \\ 1 Journals}

Édition électronique

URL : https://journals.openedition.org/crm/2456

DOI : $10.4000 / \mathrm{crm} .2456$

ISSN : 1955-2424

Éditeur

Honoré Champion

\section{Édition imprimée}

Date de publication : 15 janvier 1997

Pagination : 51-56

ISSN : 1272-9752

\section{Référence électronique}

Michel Balard, «Temps et espace du voyage chez Liutprand de Crémone », Cahiers de recherches médiévales [En ligne], 3| 1997, mis en ligne le 04 février 2008, consulté le 15 décembre 2022. URL http://journals.openedition.org/crm/2456 ; DOI : https://doi.org/10.4000/crm.2456 


\section{Temps et espace du voyage chez Liutprand de Crémone.}

Sent from the emperor Otton the Ist to the Basileus Nicephore Phocas, Liutprand of Cremona achieved two travels, a twenty years period between of them, in the constantinopolitan empire. Two antithetic perceptions of the travel according to two contrasted sensations: Liutprand of Cremona's description refers to the problem of the traveller's behaviour, often more anxious to reveal his own inside impression than a realistic description of elsewhere.

«Après quarante-neuf jours de voyage, à dos d'âne ou de cheval, à pied, affamé et assoiffé, soupirant, pleurant et gémissant, j'arrivai à Naupacte "1. De manière imagée et quelque peu agressive à l'égard de ses hôtes byzantins qui ne lui ont pas facilité la tâche, Liutprand de Crémone, envoyé de l'empereur Otton ler auprès du basileus Nicéphore Phocas, décrit son voyage de Constantinople à Naupacte, au retour de sa légation. Il nous donne en quelques lignes de précieuses indications sur la longueur des voyages entre l'Orient et l'Occident au $\mathrm{X}^{\mathrm{e}}$ siècle et sur les pays visités. Ses deux cuvres majeures, l'Antapodosis ${ }^{2}$ et la Relatio de legatione constantinopolitana, fournissent au lecteur d'aujourd'hui une image contrastée du monde byzantin, au point que l'on peut se demander si la description d'un monde si étranger ne dévoile pas davantage les préjugés et rancoeurs de Liutprand que la réalité de l'espace constantinopolitain. Le voyage vers l'Autre ne serait-il pas plutôt un voyage vers soi-même?

Les indications chronologiques fournies par l'évêque de Crémone ne manquent pas d'intérêt : l'extrême variabilité des temps du voyage est la première constatation d'évidence. En 949, Liutprand s'embarque à Pavie le $1^{\text {er }}$ août et descend le Pô jusqu'à Venise, qu'il atteint trois jours plus tard. Alors commence une première attente, partagée avec un Grec de retour d'Espagne et de Saxe et avec un riche marchand de Mayence, envoyé d'Otton. Malgré leur désir d'un prompt embarquement, il leur faut attendre jusqu'au 25 août pour quitter les bords de la Lagune, en une saison pourtant propice à la navigation et aux échanges. On ignore tout des escales et des péripéties du voyage vers Constantinople, sinon qu'il fut le plus court de tous ceux que l'on connait pour cette période: vingt-trois jours seulement de Venise aux rives de la Corne d'Or. atteintes le 17 septembre. Un temps de parcours qui correspond aux meilleures vitesses réalisées par les galères du

${ }^{1}$ Liutprand de Crémone, Relatio de legatione constantinopolitana, Scriptores rerum germanicarum, èd. E. Dümmler, Hanovre 1877. p. 161. On pourra consulter également Liutprando di Cremona, Italia e Oriente alle soglie dell'anno mille, éd. M. Oldoni P. Ariatta, Novare 1987 et Liutprand of Cremona. The Embassy to Constantinople and other writings, éd. J.J. Norwich, Londres, 1993.

2 Ed. E. Dümmler, cit.

Cahiers de Recherches Médiévales (XIIf-XV s.) , 3, 1997 
marché aux $X I V^{e}$ et $X V^{e}$ siècles ${ }^{3}$. Pour sa seconde ambassade, réalisée en 968 , Liutprand ne donne aucune indication sur son départ : il se contente de préciser qu'il arrive à Constantinople le 4 juin, vraisemblablement avec le voyage de printemps des navires vénitiens.

Mais les temps de parcours pouvaient être beaucoup plus longs, comme ce fut le cas pour le retour de la seconde ambassade, le seul sur lequel Liutprand nous donne quelques détails ${ }^{4}$. L'évêque de Crémone quitte Constantinople par bateau le 2 octobre 968. Il lui faut quarante-neuf jours pour atteindre Naupacte, sans que l'on sache pour quelles raisons il doit abandonner le navire pour utiliser ânes et chevaux. Il s'arrête trois jours à Naupacte et emprunte une barque qui le conduit au fleuve Phidari, alors que, par manque de place, ses compagnons, doivent suivre à pied le rivage. Il endure une effroyable tempête qu'il attribue à la punition de ses péchés et doit conduire sa barque jusqu'à Leucade, après la désertion des marins qui la dirigeaient. Il y parvient le 6 décembre et reçoit de l'évêque du lieu - un eunuque la plus désagréable hospitalité ; il y passe une semaine et ne reprend la mer que pour gagner Corfou en quatre jours. Le 18 décembre, nouvelle épreuve: un terrible tremblement de terre avec trois secousses successives, et, quatre jours plus tard, une éclipse de soleil qui achève de démoraliser les voyageurs. Enfin, Liutprand se trouve retenu pendant vingt jours, sans doute sur ordre d'un chambellan de Constantinople. La mesquinerie, la pingrerie et la mauvaise volonté des agents impériaux lui font prononcer plus d'un juron, aggravent les dangers du voyage et les frais supportés sur la route du retour vers la cour ottonienne. Au total, un périple de plus de trois mois, entrecoupé de tous les aléas des voyages au Moyen Age: insécurité, tempêtes, longues attentes dans les ports, manque de moyens navals appropriés, détention des voyageurs par les autorités locales, tout contribue à faire du voyage un cauchemar auquel notre ambassadeur échappe à grande peine.

Il sortait d'un autre mauvais rêve, son séjour dans la capitale byzantine, qui s'est prolongé dans la réclusion et le vide d'un palais austère du 4 juin au 20 octobre 968 : cent vingt jours d'un temps qui lui parut une éternité. Au début, les événements s'accumulent. L'évêque de Crémone arrive à Constantinople le 4 juin. Il ne reçoit pas les honneurs qu'il estime dus à son rang. A peine a-t-il reconnu les lieux que, deux jours plus tard, il a un premier entretien avec Léon, chancelier et frère du basileus. Le 7 juin, jour de Pentecôte, il est convié devant l'empereur, et après une première dispute portant sur le titre impérial, assiste, mais à une place qu'il juge indigne, au festin impérial. Le 9 juin. Liutprand tombe malade d'indignation, de chaleur et de soif. Il se plaint par lettre auprès du chancelier qui consent à le recevoir le 13 juin. Puis, le jour de la fête des Saints Apôtres, l'empereur le reçoit à nouveau à sa table en même temps que les envoyés des Bulgares. Toujours malade, il apprécie peu l'ordre de préséance du repas. Nouvelle entrevue le 21 juin, tout aussi houleuse et infructueuse que les précédentes.

${ }^{3}$ D. Stockly, Le système de l'Incanto des galées du marché de Venise (fin XIIT'-milieu XV" siècle), Leyde, 1995, p. 116.

${ }^{4}$ Liutprand de Crémone, Relatio de legatione. cit.. pp. 161-166. 
Puis le temps se traîne. Pendant trois semaines, Liutprand est confiné dans son palais inhospitalier, d'où ne peut sortir qu'un de ses domestiques préposé aux approvisionnements. Aucune visite, aucune conversation, sinon avec sa propre suite, ne vient égayer sa réclusion. Il a tout loisir de méditer sur ce qui oppose les deux empires, leur revendication exclusive du titre impérial et leurs intérêts territoriaux opposés. L'évêque se demande s'il sera un jour autorisé à rejoindre son maître, Otton. Dans la suffocation estivale, les journées lui semblent interminables et la soif insupportable.

Le 10 juillet, commence un nouveau temps de tractations. Le basileus convoque Liutprand au lieu-dit Les Fontaines, dans les faubourgs de Constantinople. La discussion achoppe une fois de plus, lorsque l'on aborde la situation des princes de Capoue et de Bénévent. Nouvelle rencontre le 20 juillet ; à sa demande, le basileus s'engage à laisser repartir Liutprand en Occident, mais le confine à nouveau dans son palais inhospitalier, d'où le tire au bout de cinq jours le frère de l'empereur pour un entretien, toujours infructueux. Le 27 juillet, enfin, l'évêque de Crémone est convoqué à nouveau, mais cette fois à Selymbria où se trouve Nicéphore Phocas. Bien que la discussion ait derechef achoppé sur la question des princes de Capoue et de Bénévent, Liutprand est retenu à la table impériale et peut admirer les bêtes sauvages que l'empereur a réunies dans un zoo. Il obtient la permission de quitter Constantinople, mais la menace sarrasine fait différer la réalisation de cette promesse impériale, d'autant que le basileus quitte alors Constantinople pour l'Orient.

Dans ces conditions, l'attente du départ devait être interminable pour l'évêque de Crémone, nul officier impérial n'osant prendre la responsabilité de lui donner congé. Trois événements seulement jalonnent les neuf semaines d'attente qui précèdent encore le départ. Le 15 août, une ambassade pontificale vient aggraver les affres de Liutprand. Contrairement aux usages. les envoyés de Rome s'adressent au basileus en le qualifiant d'empereur des Grecs. Ils sont aussitôt jetés en prison et l'évêque de Crémone craint que leur sort ne rejaillisse sur le sien. Le 14 septembre, il est autorisé à vénérer la croix et reçoit des mots de consolation de la part d'inconnus compatissants. Trois jours plus tard. il est à nouveau convoqué au palais par le patrice Nicéphore; des officiers lui confisquent en une scène célèbre les cinq coupons de pourpre qu'il voulait rapporter en Occident. Enfin, le 2 octobre, sans avoir revu le basileus, mais muni d'un lettre impériale, Liutprand est autorisé à s'embarquer.

Le temps de l'ambassade est donc un temps de pleurs et de lamentations, marqué par la maladie, la famine dont Liutprand subit en juillet les conséquences, et l'enfermement. C'est aussi un temps de polémique. où la description cède la place aux discours, toujours répétés, aucune des deux parties ne semblant vouloir céder sur l'essentiel : le titre impérial et la revendication d'un pouvoir universel. Mais, à bien regarder les choses, la rancœur de Liutprand envers les Grecs, son sentiment exacerbé de l'échec transforment en affrontement stérile un séjour diplomatique tout à fait comparable par sa longueur à celui d'autres légations. Jean de Gorze, par 
exemple, a été retenu trois ans à Cordoue ${ }^{5}$, et les envoyés d'Abd ar-Rahman III, conduits par l'évêque Recemond d'Elvira auprès d'Otton $1^{\text {er }}$, ont passé autant de temps en Germanie ${ }^{6}$. Patience et habileté sont indispensables dans des négociations dangereuses qui peuvent mettre en jeu la vie de l'envoyé. Liutprand semble avoir singulièrement manqué de ces deux qualités.

A-t-il seulement une acuité visuelle exceptionnelle, lui permettant d'apprehender rapidement l'espace et d'en donner à son lecteur une évocation éclatante? Les seules vraies descriptions que comporte le récit de ses deux ambassades portent sur Constantinople, ville impériale à double visage, selon les deux perceptions antithétiques de l'auteur : dans l'Antapodosis, la capitale splendide d'un grand empire, tête de la Chrétienté : dans la Legatio, le repère hideux d'un ennemi implacable, s'opposant aux droits légitimes du nouvel empereur germanique. Les descriptions des demeures et de la table impériale, des processions et des visites du basileus dans sa capitale portent la marque de ces contrastes.

En 949, le récit de Liutprand évoque une ville policée, où tout n'est qu'ordre et beauté. Suivons l'empereur Léon VI dans la visite incognito de sa capitale. Une nuit, le basileus décide de quitter seul, anonymement, le palais impérial et d'errer dans les rues de la ville, pour tester la fidélité et le sens du devoir de ses gardes de nuit. Arrêté comme vagabond à un premier poste de police, il allait être battu, lorsqu'il tend à ses gardes douze pièces d'or. Il est aussitôt libéré. Il subit le même sort à un second poste et s'échappe en remettant vingt pièces d'or. Au troisième, les soldats lui prennent tout ce qu'il porte et l'enferment dans une cellule. Il engage la conversation et demande à ses gardes s'ils connaissent l'empereur. "Comment pourrais-je le connaitre, répond l'un d'eux, alors que je ne me souviens même pas l'avoir jamais rencontré ". Léon VI leur dit qu'il est l'empereur, mais personne ne le croit. Après avoir discuté d'astrologie, il offre à l'un des gardes de l'accompagner au matin jusqu'au palais impérial. Reconnu par les courtisans, il donne une forte récompense aux gardes du troisième poste, mais punit et démet les autres de leur charge ${ }^{7}$.

Cette histoire impressionne fort notre évêque, mais pas autant que sa première réception au palais de Constantin VII Porphyrogénète. Le trône d'or, les lions de bronze ou de bois doré qui rugissent et agitent leur queue en cadence, l'arbre doré empli d'oiseaux qui chantent, et finalement le trône qui, à la fin de l'entrevue, s'élève dans les airs en soustrayant l'empereur aux regards des visiteurs abasourdis, tous ces symboles de la richesse de Byzance étaient là pour frapper les visiteurs étrangers et leur laisser entrevoir l'immense puissance et l'opulence de l'Empire $^{8}$. Après le palais de la Magnaure, Liutprand est ébloui par le Tribunal des XIX Lits, où pour le banquet de Noël, l'empereur et ses invités s'allongent sur les lits

${ }^{5}$ Vita Iohaniis Gorziensis, dans MGH, SS, t. IV. pp. 375-376. La comparaison entre le sort de Liutprand et celui de Jean de Gorze, dont le biographe exalte la constantia face au calife, est faite par K.J. Leyser, "Ends and Means in Liutprand of Cremona", dans Byzantinische Forschungen, t. 13, 1988, p. 131.

Ibidem, p. 128.

7 Antapodosis, éd. E. Dummler cit., livre 1, 11, pp. 7-10.

Ibidem, livre 6, 5. 
d'apparat, disposent de vaisselle d'or et prennent des fruits dans d'immenses cratères que des treuils viennent disposer devant eux ${ }^{9}$.

Cet espace de grandeur et d'opulence s'oppose à la vision que nous livre Liutprand lors de sa visite auprès de Nicéphore Phocas : une capitale aussi laide que l'est son prince, un dépouillement austère et triste. pour ne pas dire lugubre, quand il s'agit du «palais " où sont hébergés l'évêque et ses compagnons. Celui-ci, bien que de marbre, est grand ouvert aux courants d'air, ne protège ni de l'eau, ni de la chaleur, ni du froid. La couche y est de marbre et l'oreiller de pierre; l'eau potable manque et, tels des lions, des sbires retiennent prisonniers les envoyés d'Otton.

Le palais impérial ne vaut pas mieux. Là où Liutprand avait été ébloui par le faste des réceptions en 949 , il ne voit vingt ans plus tard que laideur et mesquinerie. La table impériale devient un espace long, sans largeur, et pratiquement dépouillé de tout ornement. L'évêque de Crémone s'indigne de devoir manger sur une table sans nappe. Des mets repoussants, baignés d'huile ou de garum remplacent l'abondance des fruits offerts jadis aux convives. Ail, oignon et poireau accommodent le poisson ou la viande trop grasse. Le vin, mélangé à la poix et au plâtre, est imbuvable de même que l'eau. Bref, la splendeur du décor palatial est oubliée au profit de remarques amères sur la nourriture et la boisson, auxquelles un étranger au monde byzantin ne peut $s^{\prime}$ habituer ${ }^{10}$.

Une des manifestations les plus éclatantes de l'ordre (taxis) impérial est constituée par les processions de dignitaires qui viennent rendre hommage au basileus ou l'accompagnent au cours de ses déplacements. Ici encore s'opposent deux descriptions de l'espace occupé par ces liturgies impériales. Le dernier chapitre de l'Antapodosis met un point d'orgue à la description du faste de la cour en 949 avec la "parade des gages" ", la scène du paiement des officiers, le jour des Rameaux. On voit la grande table couverte de sacs de monnaies d'or et de vêtements d'apparat, la lente procession des dignitaires et des officiers qui se présentent devant l'empereur pour recevoir selon leur rang, étoffes de soie et bourses d'or. Liutprand est lui-même honoré d'un grand vêtement de soie et d'une livre de sous d'or; par ces cadeaux, il devient en quelque sorte un client, un obligé de la cour macédonienne; il partage avec les officiers de la cour la vénération due à la personne de l'empereur. La cérémonie dure trois jours: une lenteur qui concourt avec l'ordre (taxis) fixé par l'étiquette à souligner la grandeur de l'Empire ${ }^{12}$.

Quelle déchéance en 968 pour l'envoyé d'Otton! La procession qui conduit Nicéphore Phocas du palais à Sainte-Sophie prend un tour misérable ${ }^{13}$. Ce ne sont plus des dignitaires en tenue d'apparat qui accueillent le basileus à chacune des "stations", délimitées au sol, selon la coutume, par un cercle de pourpre, mais une masse de gens de petite extraction, marchands et artisans, certains venus pieds nus,

\footnotetext{
${ }^{9}$ Ibidem, livre 6, 8.

${ }^{10}$ Sur les aliments dans le discours de Liutprand. voir J. Koder - Th. Weber, Liutprand von Cremona in Konstantinopel, Vienne, 1980.

"L'expression est de K.J. Leyser, « Ends and means ". cit.. p. 123.

${ }_{12}$ Antapodosis, éd E.Dümmer, cit, chap. 9.

${ }^{13}$ Relatio de legatione, éd. E. Dümmler, cit., chap. 9.
} 
qui constituent comme un mur de chaque côté du cortège. Les "nobles" qui participent à la procession sont vêtus de tuniques trop amples et si usagées que des trous y apparaissent, pauvre troupe déchue d'un empire décadent. Seul le basileus porte des joyaux et des ornements d'or, mais ces symboles du pouvoir font triste figure à côté des regalia de la dynastie ottonienne. Liutprand, spectateur de la procession, l'est aussi de la liturgie impériale, puisqu'il est admis aux tribunes de Sainte-Sophie, au milieu des choristes. Mais la majesté de la coupole et les ors des mosaïques ne suscitent en lui aucune admiration.

A vingt ans d'intervalle, ce sont donc deux perceptions antithétiques de I'espace constantinopolitain que nous présentent les œuvres de Liutprand: dans l'Antapodosis, la peinture de la capitale splendide d'un grand empire; dans la Relatio, celle de la ville invivable de Nicéphore. De 949 à 968 , Constantinople n'a pourtant pas changé. Ce qui a changé, c'est l'engagement personnel de l'auteur, sa haute conscience autobiographique, qui fausse son jugement. En 949, Constantinople est pour lui le centre de gravité du monde chrétien. L'Empire est à la tête de la chrétienté, et les autres Etats, Rome. l'Italie, la Francie orientale et le royaume de Bourgogne ne sont que les parties d'un tout, l'Europe, conduite par Constantinople. En 968, le centre du monde est passé à Rome et à la cour ottonienne. Liutprand a trouvé refuge dès 950 auprès d'Otton, devenu par la renovatio imperii de 962 le chef incontesté de la chrétientéts ${ }^{\prime 4}$. Le fait que le basileus refuse de reconnaître l'Empire romain d'Occident conduit l'évêque de Crémone à durcir les oppositions, en les envisageant non d'un point de vue politique et religieux, mais sur le plan des comportements et des milieux de vie.

Les écrits de Liutprand présentent donc deux Byzance opposées, en raison de deux impressions contrastées, de deux manières de juger le monde et la société, à deux moments autobiographiques différents. Liutprand s'approprie non seulement le temps, partagé entre l'admiration, l'attente et la rancoeur, mais aussi l'espace de la capitale byzantine, qu'il peuple de marionnettes pitoyables, ses contemporains, somptueusement mis en scène, avec une science parfaite des effets de théâtre ${ }^{15}$. Aussi faut-il sans doute chercher dans ses descriptions antithétiques de l'espace constantinopolitain moins l'image d'une réalité urbaine et sociale, que le reflet du paysage intérieur de l'évêque de Crémone. juge impitoyable des autres, et qui trouve son salut dans une ironie féconde et dans la certitude de l'éternité de la culture.

\section{Michel Balard (Université de Paris I)}

${ }_{14}^{14}$ R. Folz, L'idée d'empire en Occident du $V$ au XIV siècle. Paris, 1953, pp. 49-63.

15 Voir les remarques de M. Oldoni, "Liutprando oltre il magazzino delle maschere ", dans Liutprand di Cremona, Italia e Oriente alle soglie dell'anno mille, cit., p. 17, 35. 